\title{
Letter to the Editor: Differential Killing of Normal and Cystic Fibrosis Fibroblasts by Dexamethasone
}

\author{
J. EPSTEIN AND JAN L. BRESLOW
}

Division of Metabolism, Children's Hospital Medical Center, and Department of Pediatrics, Harvard Medical School, Boston, Massachusetts, USA

In a recent review, "Cystic Fibrosis at Forty-Quo Vadis," Davis and DiSant'Agnese (2) provided an excellent and comprehensive summary of the present knowledge and understanding of cystic fibrosis. However, it was stated in this review that some of our own work showing differential killing of normal and cystic fibrosis fibroblasts by dexamethasone had failed to be replicated. Although one report has indeed been published to this effect (4), we would like to point out that the authors of this report failed in several critical aspects to understand and apply our methods as we have detailed in our recently published response to that report (1). This failure to carefully reproduce our methods no doubt resulted in their obtaining results different from our own. Furthermore, we also referenced in our recent report (1) the work of two other laboratories which have reported results similar to ours when using a careful application of our published procedures (3, 5).

We would also like to report that we recently performed experiments with eight cultures of either normal or cystic fibrosis

Copyright $(1980$ International Pediatric Research Foundation, Inc. $0031-3998 / 80 / 1408-0964 \$ 02.00 / 0$ fibroblasts provided to us with blind code identification by the laboratory of Dr. Richard Erbe. Using our published techniques, we were able to correctly identify with $100 \%$ accuracy whether or not each of these cultures was from a normal or cystic fibrosis patient. This is a further demonstration that our methods are reliable when properly utilized. We hope that our work will eventually lead to a more definitive understanding of the defect in cystic fibrosis.

\section{REFERENCES AND NOTES}

1. Breslow, J. L.,and Epstein, J.: Science (Wash. D.C.). 207: 1007 (1980).

2. Davis, P. B., and DiSant Agnese. P. A.: A review. Cystic fibrosis at forty-quo vadis? Pediatr. Res., 14: 83 (1980).

3. Hallberg. T. K., Gibbs. G. E., and Acquazzino. D.: Cystic Fibrosis Club Abstracts 21st Annual Meeting, San Antonio. TX, 21: 61 (1980).

4. Kurz, J. B., Perkins, J. P.. and Buchwald, M.: Science (Wash. D.C.), 206: 1317 (1979).

5. Weichselbaum, R. W., Little, J. B., Nove. S., Hellman, S., and Piro, A. J.: J. Cell Phys. (in press) 